\title{
Effect of Lens Capsular Tension Ring on Preventing Capsular Contraction Syndrome in the Surgery of Retinitis Pigmentosa Combined with Cataract: Retrospective Case Series
}

\author{
Chang Xi Chen ${ }^{1}$, Jin Da Wang ${ }^{1}$, Jing Shang Zhang ${ }^{1}$, Ying Xiong ${ }^{2}$, Jing Li $^{2}$, Shu Ying \\ Chen $^{1}$, Zhen Yu Liu ${ }^{1}$, Xiu Li Sun ${ }^{1}$, Yusufu Mayinuer ${ }^{1}$, and Xiu Wan ${ }^{1}$ \\ ${ }^{1}$ Beijing Institute of Ophthalmology \\ ${ }^{2}$ Beijing Tongren Hospital
}

June 22, 2020

\begin{abstract}
PURPOSE: To observe the effect of phacoemulsification and intraocular lens (IOL) implantation with or without lens capsular tension ring (CTR) on retinitis pigmentosa (RP) combined with cataract patients. DESIGN: Retrospective cases series study. METHODS: 63cases (84 eyes) of RP with cataract were collected including 30 males and 33 females. Phacoemulsification with $3.0 \mathrm{~mm}$ clear corneal incision was performed in all the patients. IOL+CTR implantation was performed in 44 eyes, and only IOL implantation was performed in 40 eyes. All cases were followed up at 1 week and 1, 3, 6 months after the surgery to compare the best corrected visual acuity (BCVA), intraocular pressure (IOP), corneal endothelial cell count and complications before and after the surgery. RESULTS: all surgery were successfully completed by the same physician, and IOL and CTR were all implanted in capsule without complications. The BCVA at 6 months after surgery was $0.91 \pm 0.88$ LogMAR. It improved a little compared with the BCVA $(1.3 \pm 0.7 \log M A R)$ before surgery and there was a signifcant difference in statistic $(\mathrm{P}=0.003)$. Four cases of capsule contraction syndrome $(\mathrm{CCS})$ occurred in no CTR implantation group and there was no CCS in CTR group. There was signifcant difference in statistic about the incidence of CCS between two groups $(\mathrm{P}=0.047)$. CONCLUSIONS: Phacoemulsification for RP combined with cataract is safe and reliable, and CTR implantation is helpful to reduce the complications caused by capsule contraction.
\end{abstract}

\section{Background:}

Retinitis pigmentosa (RP) is a clinically common congenital retinal disease, with a incidence of about $1 / 4000^{1}$. The pathological manifestations include progressive dysfunction of retinal pigment epithelial cell and photoreceptor cell, and patients tend to be night blind, have reduced vision field and poor vision. Currently, there is no effective treatment or prevention measures for RP in world, and such as stem cell transplantation and gene therapy are still in the research stage ${ }^{2}$. In addition to primary retinal damage, RP is usually accompanied by other eye diseases. Cataract is the most common complication of RP. Patients with RP complicated with cataract are young in age and progress rapidly, which has a great impact on the remaining vision of patients, and usually need surgical treatment. This paper retrospectively analyzed the vision, intraocular pressure, corneal endothelial cell count, macular morphology and postoperative complications of 63 patients (84 eyes) who underwent phacoemulsification and IOL implantation in our hospital from January 2014 to October 2019. The results are as follows.

\section{Methods}

Subjects 
The study complied with the tenets of the Declaration of Helsinki and was approved by the Ethics Board of the Beijing Tongren Hospital. Written informed consent was obtained from all patients before surgery. A total of 63 cases (84 eyes) of RP combined with cataract treated in our hospital from January 2014 to October 2019 were collected, including 30 males and 33 females, aged 35-58 years, with an average age of 42.8 years. Phacoemulsification with a $3.0 \mathrm{~mm}$ clear corneal incision was performed in all the patients. IOL + capsular tension ring (CTR) implantation was performed in 44 eyes and only IOL implantation was performed in 40 eyes. All the collected cases were RP patients with cataract, no other eye diseases, no history of eye surgery or trauma.

\section{Examinations}

Before surgery, all the enrolled patients were examined by slit lamp, intraocular pressure(IOP) and corneal endothelial cell count(ECC) were measured. All patients were taken refractive examination and the best corrected vision(BCVA) was determined. Corneal curvature was determined by keratometer and ABultrasonography were performed to determine the axial length of the eye. SRK-T formula was used to calculate the IOL degree. Fundus photos were taken and OCT was used to examine fundus conditions .Follow-up visits were performed 1 week, 1 month, 3 months and 6 months after the operation, and all preoperative examinations were also performed to evaluate postoperative recovery and complications.

\section{Surgery}

A single surgeon ( Wan Xiu Hua) performed standard phacoemulsification and IOL implantation in all cases. One hour before the operation, tropicamide eye drops was applied to ensure the pupil dilation and ciliary muscle relaxation during the operation. The conjunctival sac was anesthetized twice within 5-10 minutes before surgery. A $3.0 \mathrm{~mm}$ corneal incision was made in the transparent area of the limbal cornea between 9 to 12 points and an auxiliary incision was made at 2 points of the corneal limbus. The viscoelastic agent was injected into the anterior chamber, Continuous circular capsulorhexis was performed, water separation nucleus and surrounding cortex appeared to the "golden ring". Phacoemulsification removed lens nuclear and removed cortex.The anterior chamber and capsule were filled with viscoelastic agent. The CTR and IOL were implanted through $3.0 \mathrm{~mm}$ incision. The viscoelastic agent was removed from the anterior chamber and the incision was watertight.

\section{Statistical analysis}

Statistical analysis was performed using SPSS for Windows (version 22.0; IBM-SPSS, Chicago, IL, USA). Paired t-test was used to compare preoperative and postoperative BCVA, IOP, and corneal ECC. The independent sample $t$ test and the chi-square test were used to compare the two groups. P-values represent results for 2 - sided tests, with values less than 0.05 considered statistically significant.

\section{Results}

All operations were successfully completed by the same physician, and IOL and CTR were all implanted in capsule without intraoperative complications.

The data of preoperative and postoperative BCVA, IOP, and corneal ECC were showed in table 1 . The BCVA at 6 months after surgery was $0.91 \pm 0.88$ LogMAR. It improved a little compared with the BCVA before surgery(1.3 $\pm 0.7 \operatorname{LogMAR})$, and there was a signifcant difference in statistic $(\mathrm{P}=0.003)$. There were 22 eyes which had significantly improved visual acuity, 20 eye had slightly improved visual acuity, and the others had no significant improvement. The IOP was higher and the corneal ECC was less at 6 months after surgery compare with the data before surgery and there were also signifcant differences in statistic $(\mathrm{P}=0.000)$. Bur they were both in the normal range. Oct examination showed no change of macular morphology before and after surgery.

\section{Postoperative complications}

There were four cases of capsule contraction syndrome(CCS) (Fig.1) in the group without CTR implantation at 6 months after surgery and there was no CCS in CTR group. There was signifcant difference in statistic 
about the incidence of CCS between two groups $(\mathrm{P}=0.047)$. There were no other statistically significant differences about other conditions between the two groups (table 2). All CCS cases were cured through capsule loosening surgery.

\section{Discussion}

$\mathrm{RP}$ is a common hereditary eye disease and most RP patients are complicated with cataract. The lens opacification often occurs in the central of posterior capsule or posterior polar cortex ${ }^{3}$, which seriously affects the residual tubular vision. For patients with RP complicated with cataract, current studies have proved that most patients have improved visual acuity and visual field after surgery ${ }^{4}$, and the effect is clear. Cataract surgery itself is safe and reliable, and does not lead to the occurrence and progress of other RP related complications. Therefore, it is suggested that RP complicated with cataract should be treated by surgery ${ }^{5-7}$. The results of this study also showed that most of the patients' vision was improved, the operation effect was good, and the operation was safe and reliable.

Most studies have reported that the final postoperative vision of patients is closely related to the structure of macular area ${ }^{8,9}$. In this study, it was also found that the visual acuity of patients with relatively healthy macular before surgery was significantly improved, while the visual acuity of patients with significantly thinner macular was limited. This also suggests that for RP patients with cataract, preoperative OCT examination is very important for predicting postoperative vision ${ }^{10}$.

Due to the common lens suspensory ligament relaxation in RP patients with cataract, it is necessary to pay attention to the prevention and treatment of capsule tear, suspensory ligament rupture and other related intraoperative complications. The capsule contraction of such patients is relatively rapid after surgery. The suspensory ligament relaxation is easy to cause CCS and dislocation of the IOL ${ }^{11}$. In this study, four cases of CCS occurred, and were treated with capsule loosening operation at six months after the cataract surgery. The patients were not implanted with CTR. And there was no CCS among CTR implanted patients. Bayyoud $\mathrm{T} \mathrm{etc}^{12}$. reported that there were 52 eyes of RP with cataract surgery patients. Two cases of CCS happened in no implanted CTR group, while there no CCS occured in implanted CTR group after a median followup of 26 months. The incidence was $4 \%$. It also suggested that CTR implantation can help to reduce the postoperative CCS in RP with cataract surgery. In addition, patients with RP complicated with cataract are relatively young in age, and posterior capsular opacity (PCO) occurs quickly, which requires timely treatment. CTR implantation also has certain effects on the prevention of $\mathrm{PCO}^{12}$.

Our results showed that IOP was higher and corneal ECC was less after surgery. Though they were in normal range, but it reminded us to pay attention to the IOP and ECC in RP with cataract surgery. There were some reports that the postoperative macular edema may occur in RP with cataract surgery ${ }^{5,12}$. But there was not found in this study. The occurrence of postoperative macular edema of cataract surgery associated with many factors. Because of the RP patients themselves severe retinopathy, macular edema would further damage eyesight. So you need to pay special attention to that postoperative oct examination should be carried out regularly .

\section{Financial Disclosure}

No authors have a financial and proprietary interest in any material and method mentioned.

\section{References}

1. Verbakel SK, van Huet R, Boon C, den Hollander AI, Collin R, Klaver C, et al. Non-syndromic retinitis pigmentosa. Prog Retin Eye Res 2018. 66:157-186.

2. Huang Y, Enzmann V, Ildstad ST. Stem cell-based therapeutic applications in retinal degenerative diseases. Stem Cell Rev 2011. 7:434-445.

3. Auffarth GU, Tetz MR, Krastel H, Blankenagel A, Völcker HE. [Complicated cataracts in various forms of retinitis pigmentosa. Type and incidence]. Ophthalmologe 1997. 94:642-646. 
4. Chan T, Lam SC, Mohamed S, Wong R. Survival analysis of visual improvement after cataract surgery in advanced retinitis pigmentosa. Eye (Lond) 2017. 31:1747-1748.

5. Davies EC, Pineda R. Cataract surgery outcomes and complications in retinal dystrophy patients. Can J Ophthalmol 2017. 52:543-547.

6. De Rojas JO, Schuerch K, Mathews PM, Cabral T, Hazan A, Sparrow J, et al. Evaluating Structural Progression of Retinitis Pigmentosa After Cataract Surgery. Am J Ophthalmol 2017. 180:117-123.

7. Dikopf MS, Chow CC, Mieler WF, Tu EY. Cataract extraction outcomes and the prevalence of zonular insufficiency in retinitis pigmentosa. Am J Ophthalmol 2013. 156:82-88.e2.

8. Nakamura Y, Mitamura Y, Hagiwara A, Kumagai K, Miura G, Sugawara T, et al. Relationship between retinal microstructures and visual acuity after cataract surgery in patients with retinitis pigmentosa. $\mathrm{Br} \mathrm{J}$ Ophthalmol 2015. 99:508-511.

9. Sandberg MA, Brockhurst RJ, Gaudio AR, Berson EL. The association between visual acuity and central retinal thickness in retinitis pigmentosa. Invest Ophthalmol Vis Sci 2005. 46:3349-3354.

10. Yoshida N, Ikeda Y, Murakami Y, Nakatake S, Fujiwara K, Notomi S, et al. Factors affecting visual acuity after cataract surgery in patients with retinitis pigmentosa. Ophthalmology 2015. 122:903-908.

11. Ascaso FJ, Huerva V, Grzybowski A. Epidemiology, Etiology, and Prevention of Late IOL-Capsular Bag Complex Dislocation: Review of the Literature. J Ophthalmol 2015. 2015:805706.

12. Bayyoud T, Bartz-Schmidt KU, Yoeruek E. Long-term clinical results after cataract surgery with and without capsular tension ring in patients with retinitis pigmentosa: a retrospective study. BMJ Open 2013. 3 .

Figure legend

Figure 1: The cases of the lens' capsule contraction syndrome(CCS) .

\section{Hosted file}

table1.doc available at https://authorea.com/users/335583/articles/461458-effect-of-lenscapsular-tension-ring-on-preventing-capsular-contraction-syndrome-in-the-surgery-ofretinitis-pigmentosa-combined-with-cataract-retrospective-case-series

\section{Hosted file}

table2.doc available at https://authorea.com/users/335583/articles/461458-effect-of-lenscapsular-tension-ring-on-preventing-capsular-contraction-syndrome-in-the-surgery-ofretinitis-pigmentosa-combined-with-cataract-retrospective-case-series

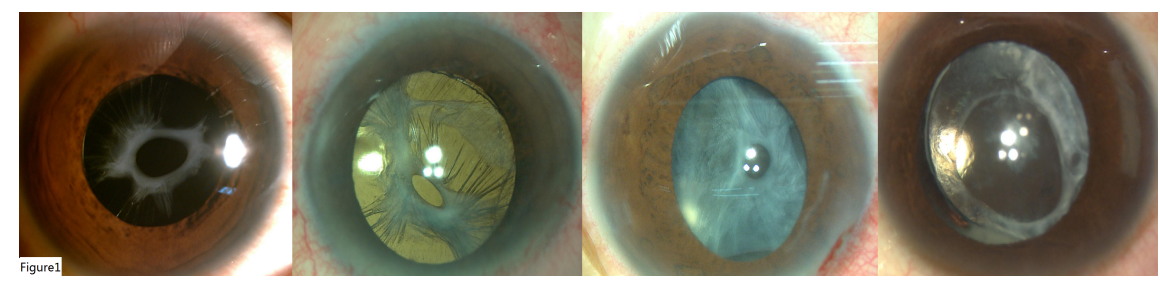

